\title{
Prognosis of Thoracic Cancer Using the Bierman Random Committee Machine Learning
}

\author{
https://doi.org/10.3991/ijoe.v17i12.27573 \\ Ezzat A. Mansour(网) \\ King Abdulaziz University, Jeddah, Saudi Arabia \\ ezzat556655@hotmail.com
}

\begin{abstract}
Thoracic most cancers are a prime problem in the clinical field. Unexpected occurring cannot be predicted earlier but if the strategy is fine-tuned properly then the prognosis of cancer is not a major issue. But the problem is how to find out the proper layout with all possible features. The sector of Thoracic Surgery is offering a source of the dataset with all feasible attributes of thoracic cancer. All the features suggested by this medical sector were approved by the Consortium of Tuberculosis and Pulmonary Diseases. The random committee is a novel hybrid algorithm that utilizes the benefit of both random forests with committee concepts. Many random forests are created as the result of the iteration. But anyone can be created and the committee analyses and retains any one optimal solution. Breiman, the first researcher to propose the general concept of Radio Frequency following the same he proposed the famous and most popular forest RF algorithm.
\end{abstract}

Keywords - thoracic cancer, random committee forest, machine learning algorithm

\section{Introduction}

Worldwide thoracic cancer accounts for $15.6 \%$ of general most cancers instances; it's miles for the maximum element of the leading reason of cancer dying. Although there is a superb boom in era the analysis of thoracic most cancers ruin bad. Artificial intelligence era and the arrival of comics, which encompass radiomics, proteomics, genomics, and transcriptomic multimers evaluation primarily based on system gaining has an excellent capability to enhance thoracic most cancers evaluation [1].

Maximum cancers may be amendable and non-amendable. Amendable most cancers involve behavioral and well-being habits which includes tobacco use, weight gain and plenty of others and non-amendable genetic elements consisting of genetic mutations inherited and immune deficiencies. Smoking is the number one hazard element for lung maximum cancers, even though research display that smoking is associated with eighty $\%$ of women and sixty-nine $\%$ of men as in parent [2]. 


\section{Global Cancer} Incidence

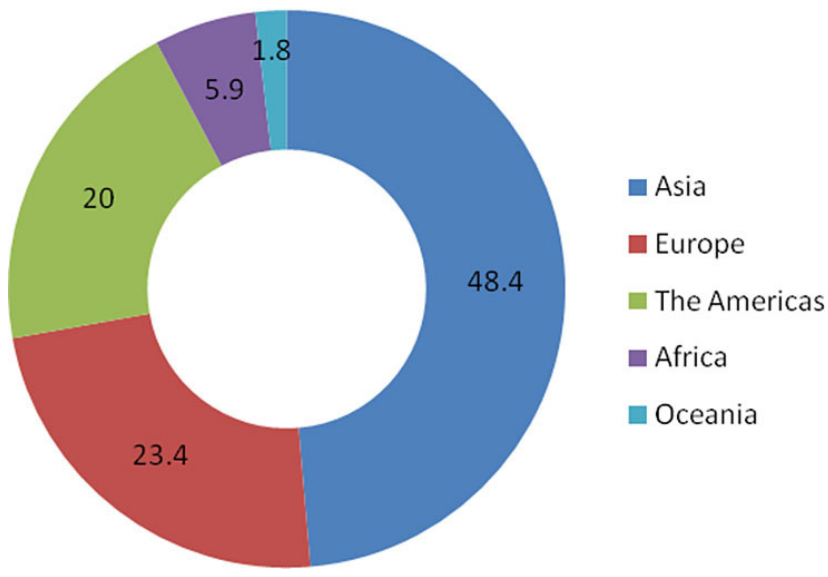

Fig. 1. Survey report 01

The concept of superior restoration after surgical treatment (ERAS) refers back to the software program application of preoperative, intra operative and put up operative strategies to lessen surgical stress, ultimately lowering the charge of headaches and accelerating patient healing. Prerecorded - enabled thoracic surgical operation has been frequently practical in thoracic surgical remedy from 1990. VATS lobotomy reasons plenty much less trauma and much less postoperative headaches as compared to standard open thoracotomy and will accelerate affected character recovery [3]. Figure 1 shows the survey report.

The implementation of minimally invasive strategies for lobar resection has been one of the vital advances in thoracic surgical operation. As a barely insistent substitute to open thoracotomy, Video-enabled thoracic surgical procedure is the precise modality in the suggestions given for early prediction. Stereotactic frame radiotherapy (SBRT) is a treatment alternative for degree I sufferers who are medically inoperable or refuse surgical procedure [4].

\section{Related work}

Now-a-days researchers are focusing on different disciplines which includes medical, biological and radiomics. Though, the appearance of the domain of radiomics, has a Connection involving biomarkers and radiomics attributes has concerned escalating explore significance [1].

Doyen Kim supplied a visible-primarily based definitely state of affairs for mixture of multiomics information and genomic information for the prophecy of clinical most cancers products. Medical and genomic data outlines for thoracic most cancers records resource from TCGA data porch be taken and studies became finished. The final results 
prove that the expected action finished exceptional with the constructive cost which become received beneath curve price is 0.7866 [2].

Medical information of 89 patients with minor symptoms of small lump or the cell in small size was admitted in the hospital affiliated to the University of Soochow between first January 2016 to the end of February 2017 and their reports are analyzed. Out of the given record 30 patients are suggested for chemotherapy and 59 patients are suggested for early surgical treatment [3].

Amusingly, approximately all machine learning algorithms used in cancer prediction and prediction utilize supervised learning. In addition, the majority of these administered learning procedures belong to an explicitly ass of classifiers that categorize on the beginning of provisional probability or provisional results [5-7].

Prior to assessment of class algorithms, it is critical to recall which comparative measure must be used. Moreover, as soon as one or numerous degree(s) have been decided on, it's miles can be handiest to anticipate the data. Consequently, finding out exactly how this overall performance measure must be anticipated, and overall performance measures have been predicted for each algorithm, differences between them can be evaluated using diverse statistical checks $[7,8]$.

\section{System architecture}

Thoracic Cancer is a critical problem in medical field. Early diagnosis and prevention is the major intention of this research work. Detection is based on the features obtained from the lung cancer data set $[9,10]$. A conventional method is proposed which integrates the gene expression data and classifies the gene expression profile as in Figure 2. The proposed conventional method may be compared with the other machine learning models to satisfy the given hypothesis statement.

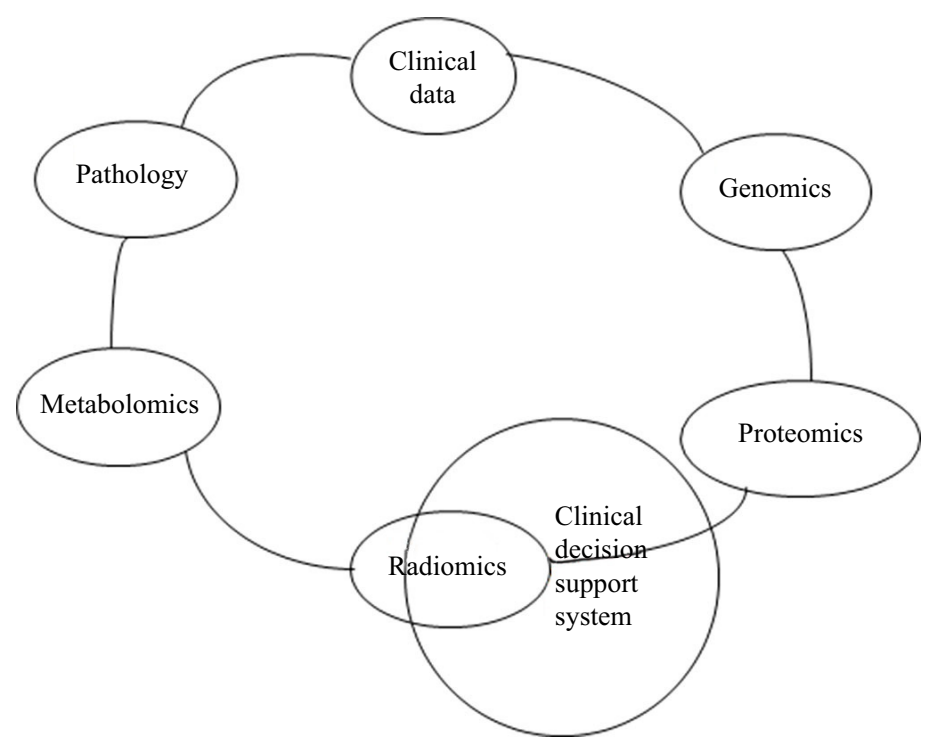

Fig. 2. Graphical representation of clinical support decision system [1] 
Pattern matching and gene discovery is done for both discrete and continuous framework model. Medical Association of India is providing simulation data set for early prognosis of cancer $[11,12]$. The researchers can use the sample of tumor patients for analyzing and doing experiments. The accuracy ratio is done and refereed for future study as in Figure 3.

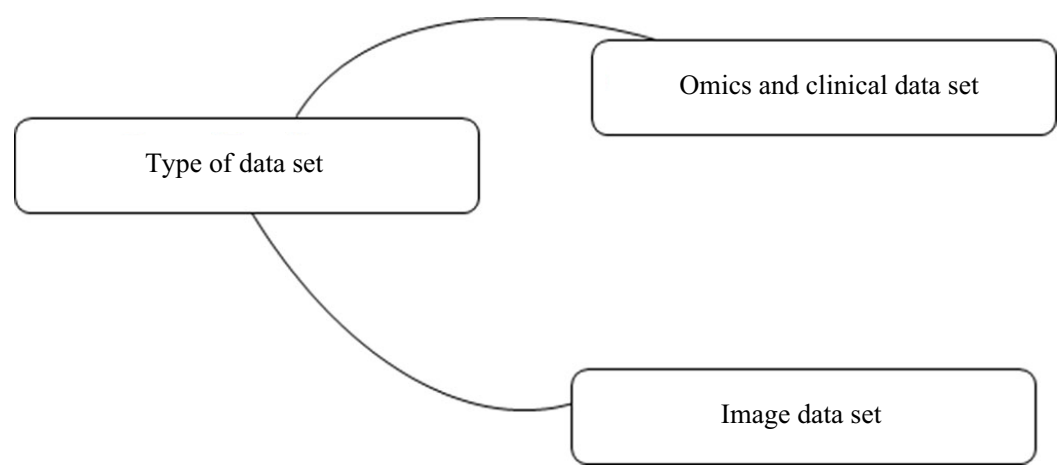

Fig. 3. Types of data used for lung cancer

\section{$4 \quad$ Proposed system}

Proposed TC_RC Algorithm is given below

1. Preprocess the Thoracic cancer dataset for missing values and misnomers

2. For each sample in the thoracic cancer dataset

3. Perform the random committee process

4. For each member in the random committee, $\mathrm{T}$

5. Select subsets and create possible $\mathrm{N}$ number of solutions

6. The selected random committee subset must be $>66 \%$

7. For each node

a. Select the predictor variable $\mathrm{m} 1$

b. The predictor value must provide the best spilt which-leads to the optimal solution

c. For each node, NODE choose another $\mathrm{m}^{2}$ samples and repeat the process again

8. For the splitting criteria three choices can be opt based on the number of samples

9. Random splitting criterion: $\mathrm{m}$ equals one

10. Breiman's bagger splitting criterion:m equals total predictor values

11. Random committee splitting criterion:m less than the total count of predictor values.

12. Brieman value for mare: $1 / 2 \sqrt{\mathrm{m}}, \sqrt{\mathrm{m}}$, and $2 \sqrt{\mathrm{m}}$ 


\subsection{Data set information}

The dataset is related to the branch of thoracic surgical treatment. Table 1 shows the attribute information for experimental results. Figure 4 depicts single decision tree, gradient boosted trees and random forest while Figure 5 demonstrates Bootstrap Sampling, Building the models \& Bootstrap Aggregating.

Table 1. Attribute information

\begin{tabular}{|c|}
\hline $\begin{array}{l}\text { 1. DGN-Analysis of ICD-10 codes for primary and secondary as well more than one tumors' if any } \\
\text { (DGN3, DGN2, DGN4, DGN6, DGN5, DGN8, DGN1) }\end{array}$ \\
\hline 2. PRE4-Forced vital capability-FVC (numeric) \\
\hline $\begin{array}{l}\text { 3. PRE5-quantity that has been exhaled at the end of the first second of compelled } \\
\text { expiration-FEV1 (numeric) }\end{array}$ \\
\hline 4. PRE6 - Overall result (performance) repute-Zubrodscale (PRZ2, PRZ1, PRZ0) \\
\hline 5. PRE7—Pain before surgical procedure $(\mathrm{T}, \mathrm{F})$ \\
\hline 6. PRE8-Haemoptys is earlier than surgical treatment $(\mathrm{T}, \mathrm{F})$ \\
\hline 7. PRE9—Dyspnoea earlier than surgical procedure $(\mathrm{T}, \mathrm{F})$ \\
\hline 8. PRE10 — Cough earlier than surgical procedure $(\mathrm{T}, \mathrm{F})$ \\
\hline 9. PRE11—Weakness before surgical procedure $(\mathrm{T}, \mathrm{F})$ \\
\hline $\begin{array}{l}\text { 10. PRE14-T in clinical TNM - size of the unique tumour, from OC11 (smallest) to OC14 (biggest) } \\
\text { (OC11, OC14, OC12, OC13) }\end{array}$ \\
\hline 11. PRE17—Kind 2DM—diabetes mellitus (T,F) \\
\hline 12. PRE19-MI upto 6 months (T,F) \\
\hline 13. PRE25-PAD-peripheral arterial sickness (T,F) \\
\hline 14. PRE30-Smoking (T,F) \\
\hline 15. PRE32-Bronchical Asthma (T,F) \\
\hline 16. AGE-Age at surgery (numeric) \\
\hline 17. Risk1Y-1 year survival duration- $-(T)$ rue value if died $(\mathrm{T}, \mathrm{F})$ \\
\hline $\begin{array}{l}\text { Elegance Distribution—the elegance cost (Risk1Y) is binary valued. Risk1Y duration—wide variety of } \\
\text { times: T70 N } 400\end{array}$ \\
\hline
\end{tabular}

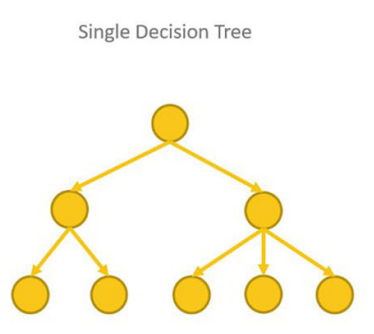

Fig. 4. Single decision tree, gradient boosted trees and random forest [2]

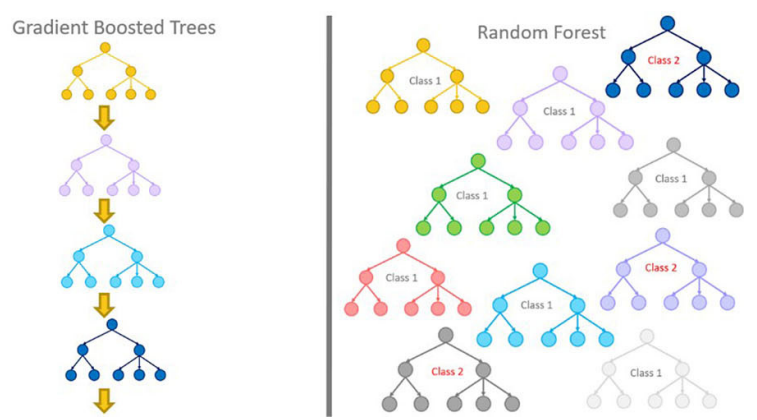




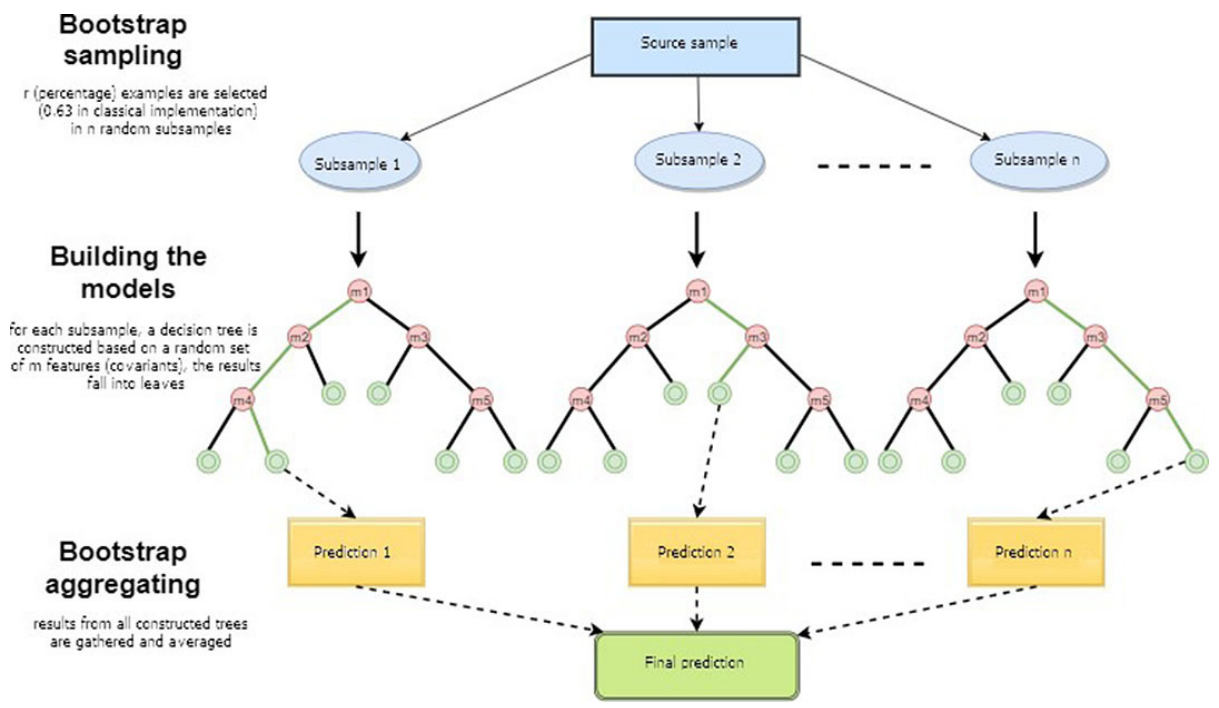

Fig. 5. Bootstrap sampling, building the models \& bootstrap aggregating [2]

\section{Work scenario}

This work was executed in Google Colab Notebook, Implemented with python, Based on precision results for different ration on database AE with LSTM. It was not a successful experience and the Classify the AE best result with Over-Sampling techunique. The workflow of random committee involves two significant traits: In the random committee, reduced bias with increased variance Reduced Variance inspite of no alteration in bias.

\subsection{Exemplification}

The hypothesis referenced above gives a conspicuous technique: make a bunch of specialists with low inclination and high difference, and afterward normal them. By and large, this means to make a bunch of specialists with changing boundaries; every now and again, these are the underlying synaptic loads, all be it different variables, (for example, the learning rate, force and so forth) might be shifted also. A few creators advise against fluctuating weight rot and early stopping. The means are along these lines:

1. Generate $\mathrm{N}$ experts, each with their personal underlying characteristics (beginning features are generally picked arbitrarily from dispersion).

2. Train every master independently.

3. Combine the specialists and normal their qualities. 
On the other hand, space information might be utilized to create a few classes of specialists. A specialist from each class is prepared, and afterward joined.

Where $\alpha$ is a group of weights. The development difficulty of coming across alpha is right away tackled via neural groups, eventually a "meta community" wherein each "neuron" is reality be informed an entire neural organization can be organized, and the synaptic masses of the remaining organization is the burden implemented to every grasp, this is called an instantaneous blend of experts. Figure 2 is based on blending experts.

$$
y(x ; \alpha)=\sum_{j=1}^{\varphi} \alpha_{j} y_{j}(x)
$$

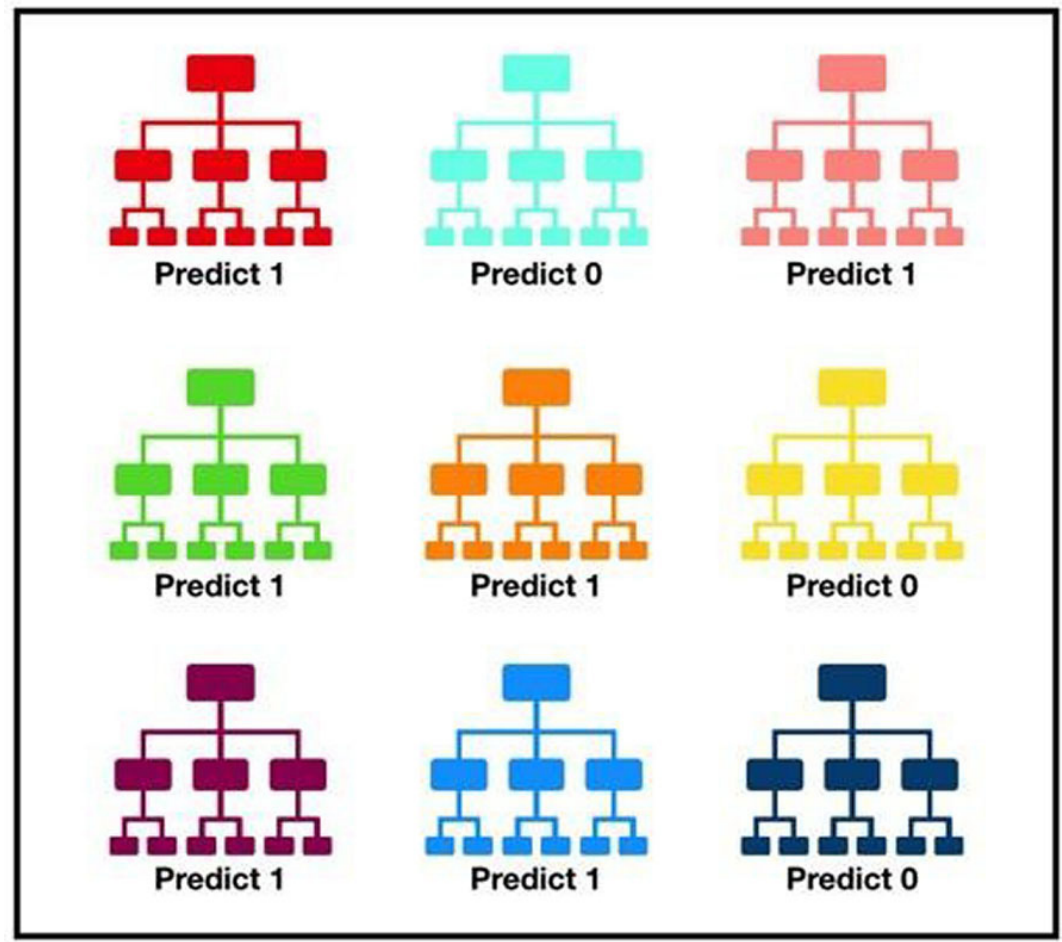

Fig. 6. Blending of experts [6]

\section{Notion of a random forest model creating a prediction}

In data era communicate, the purpose that the Random forest model functions admirably is: limitless fairly uncorrelated fashions (trees) operate as a committee will beat any of the person constituent models [13].

The low relationship amongst models is the critical issue. Very similar to how ventures with low connections (like shares and securities) join up to border a portfolio that is greater awesome than the quantity of its factors, uncorrelated fashions can create 
outfit forecasts which is probably more precise than any of the person expectancies. The reason within the returned of this top notch effect is that the timber guard every other from their person mistakes (so long as they do not normally all fail a similar manner) [14]. Whilst a few trees won't be proper, several distinctive timbers can be accurate, in order a meeting the timber can circulate the proper way. So, the necessities for Random woodland to carry out well are:

- The ensuing committee is sort of continually much less complex than a single community that could achieve the equal stage of performance.

- The ensuing committee may be skilled extra without problems on smaller input units.

- The resulting committee regularly has improved performance over any single community.

- The hazard of over becoming is lessened, as there are fewer parameters (weights) which want to be set.

\section{$7 \quad$ Technical description}

For the technical desction we used Weka.classifiers.meta.Random Committee

\subsection{Description}

Class for building ensemble base classifiers. Every base classifier is built with the use of a random wide variety seed (however based at the equal records). The final prediction is instantly common for the predictions generated by means of the character base classifiers.

\subsection{Options}

Seed-The random number seed to be used. Num Execution Slots - The number of execution slots (threads) used for constructing the ensemble.

Num Decimal Places-It is used for the output of numbers in the model. It is represented in decimal.

Batch Size-The preferred number of instances to process if batch prediction- is being performed. More or fewer instances may be provided, but this gives implementations a chance to specify a preferred batch size. Num Iterations-The iterations to be performed debug - If it is assigned to true, classifier may output additional information to the console classifier-The base classifier to be used. Figure 7 represents classifiers for building an ensemble of randomizable base classifiers.

Do Not Check Capabilities - If set, classifier capabilities are not checked before classifier is built (Use with caution to reduce runtime). 


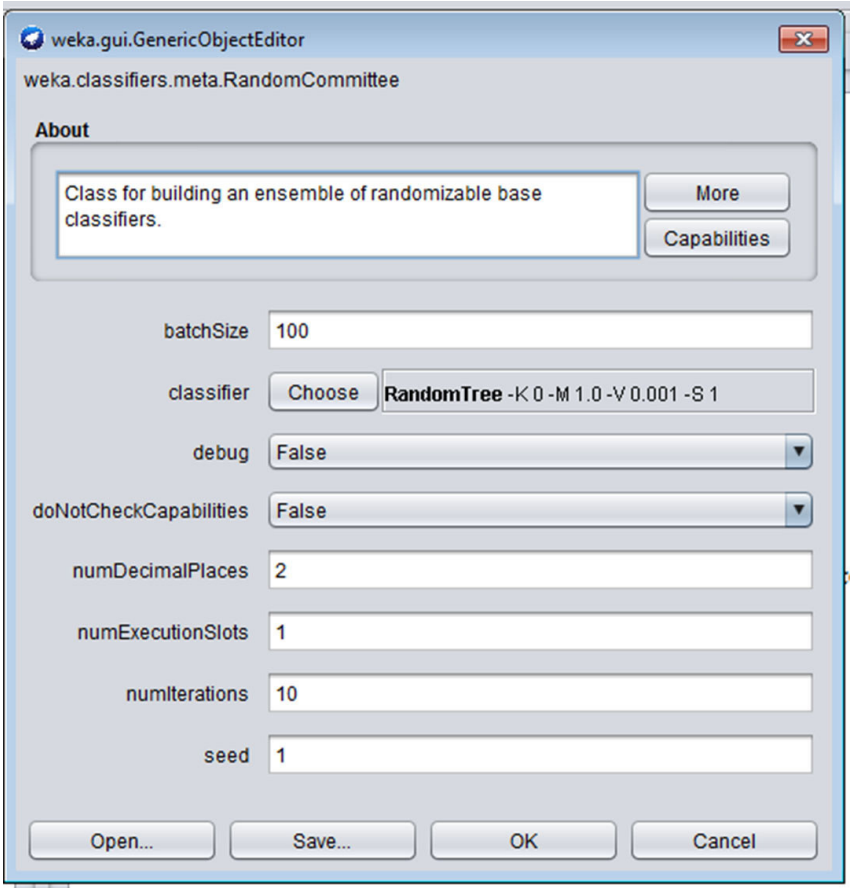

Fig. 7. Class for building an ensemble of randomizable base classifiers

\subsection{Experimental results}

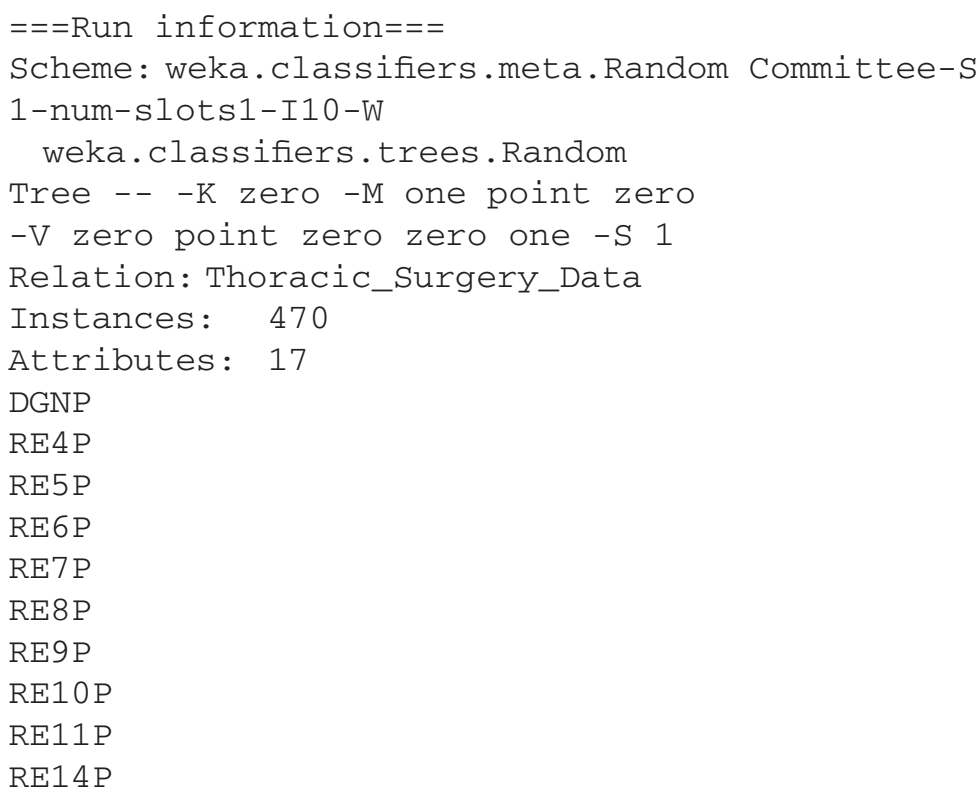




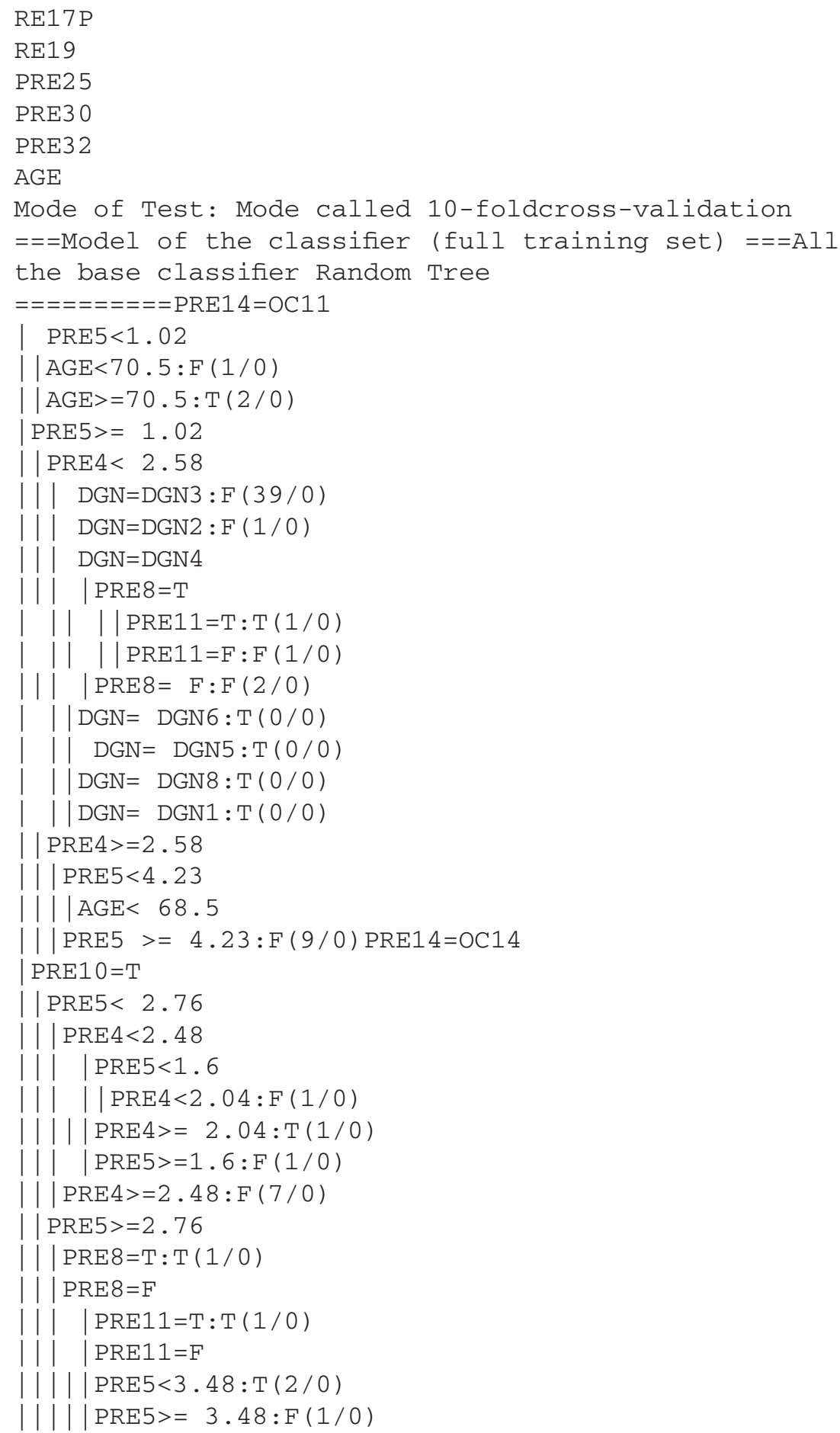




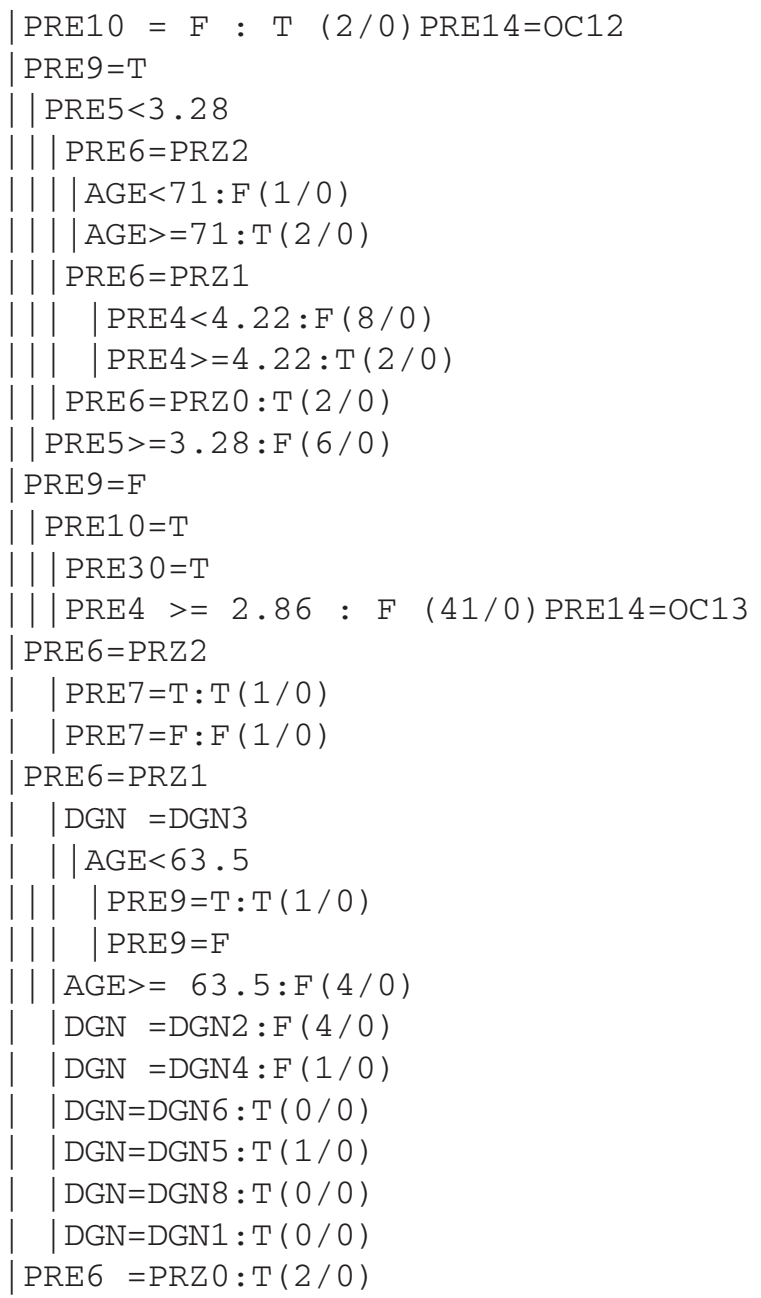

Size of the tree: 261

Time taken to build model: 0.1 seconds

The above results are compiled in Weka where size of the trees is 261 and it took only 0.1 second to build a file. Table 2 and Table 3 shows accuracy and confusion matric. 
Table 2. Accuracy by using the elegance of random forest

\begin{tabular}{|l|l|c|c|}
\hline \multicolumn{1}{|c|}{ S.No } & \multicolumn{1}{|c|}{ Mode of Instances } & Instances & \% \\
\hline 1 & Correctly Classified Instances & 372 & 79.1489 \\
\hline 2 & Incorrectly Classified Instances & 98 & 20.8511 \\
\hline 3 & Value of the Kappastatistic & 0.015 & - \\
\hline 4 & Value Mean absolute error & 0.237 & - \\
\hline 5 & Value of Root mean squared error & 0.3879 & - \\
\hline 6 & Value of the Relative absolute error & - & 93.0499 \\
\hline 7 & Value of the Root relative squared error & - & 108.94 \\
\hline 8 & Total Number of Instances & 470 & - \\
\hline
\end{tabular}

Table 3. Confusion matrix

\begin{tabular}{|l|c|c|c|c|c|c|c|c|}
\hline $\begin{array}{c}\text { True/ } \\
\text { Positive } \\
\text { Rate }\end{array}$ & $\begin{array}{c}\text { False } \\
\text { Positive } \\
\text { Rate }\end{array}$ & $\begin{array}{c}\text { Precision } \\
\text { in } \\
\text { Decimal }\end{array}$ & $\begin{array}{c}\text { Recall } \\
\text { in } \\
\text { Decimal }\end{array}$ & $\begin{array}{c}\text { F-Measure } \\
\text { in decimal } \\
\text { value }\end{array}$ & $\begin{array}{c}\text { MCC in } \\
\text { Decimal } \\
\text { Value }\end{array}$ & $\begin{array}{c}\text { ROC } \\
\text { Area in } \\
\text { Decimal } \\
\text { Value }\end{array}$ & $\begin{array}{c}\text { PRC } \\
\text { Area in } \\
\text { Decimal } \\
\text { Value }\end{array}$ & $\begin{array}{c}\text { Class } \\
\text { True/ } \\
\text { False }\end{array}$ \\
\hline 0.100 & 0.088 & 0.167 & 0.100 & 0.125 & 0.016 & 0.615 & 0.200 & $\mathrm{~T}$ \\
\hline 0.913 & 0.900 & 0.853 & 0.913 & 0.882 & 0.016 & 0.615 & 0.892 & $\mathrm{~F}$ \\
\hline $\begin{array}{l}\text { Value of the } \\
\text { Weighted } \\
\text { Average }\end{array}$ & 0.791 & 0.779 & 0.751 & 0.791 & 0.769 & 0.016 & 0.615 & 0.789 \\
\hline
\end{tabular}

\section{Performance comparison}

The results can be considered successful if the performance comparison is accurate. We measured threshold curve, margin curve, cost benefit analysis, cost curve, and agevs. level attribute distribution graph and received promising results. Figures 8-12 shows the detailed comparison of each factor discussed above in Weka classified. 


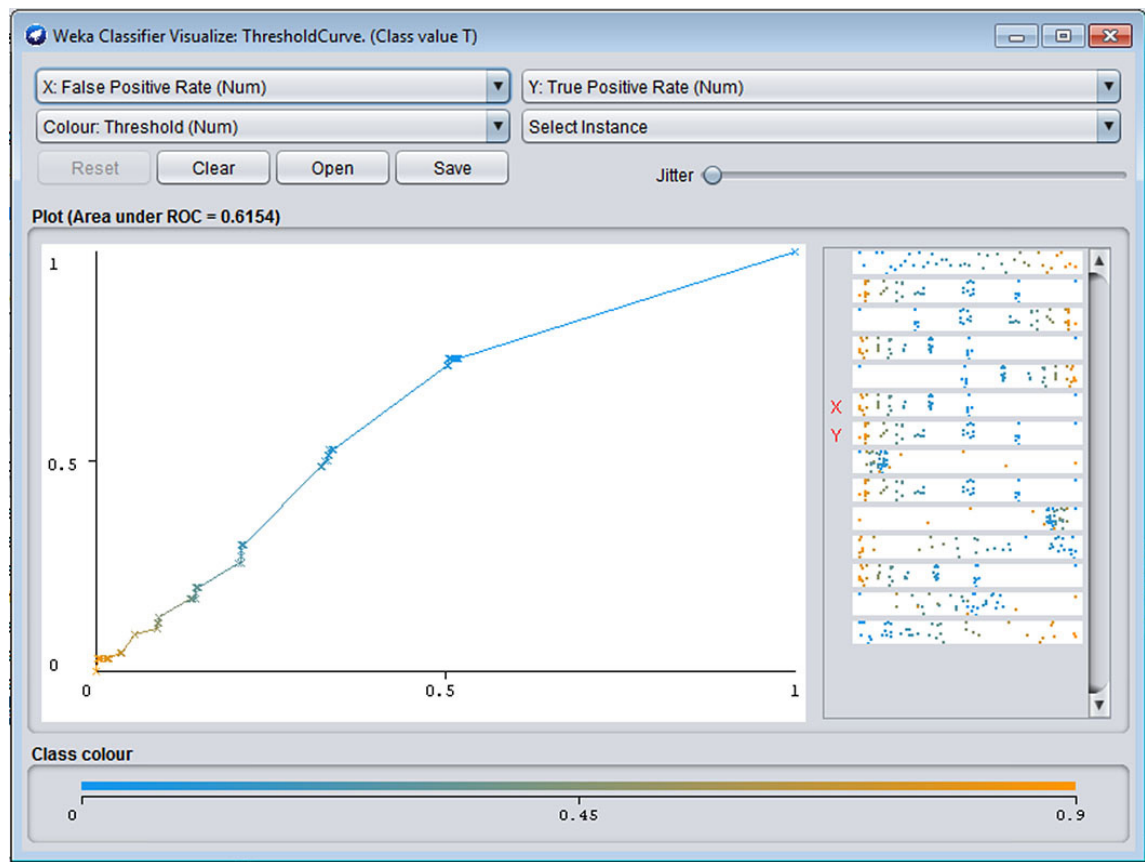

Fig. 8. Threshold curve

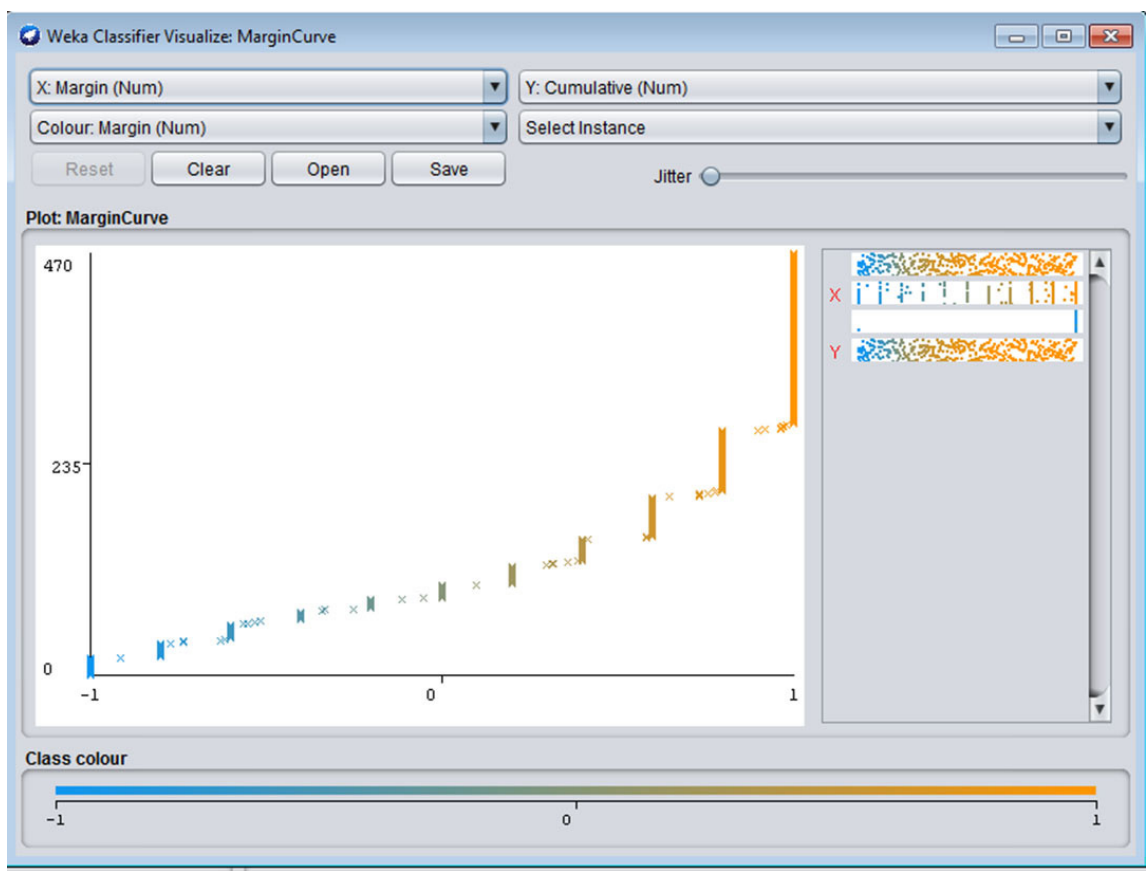

Fig. 9. Margin curve 


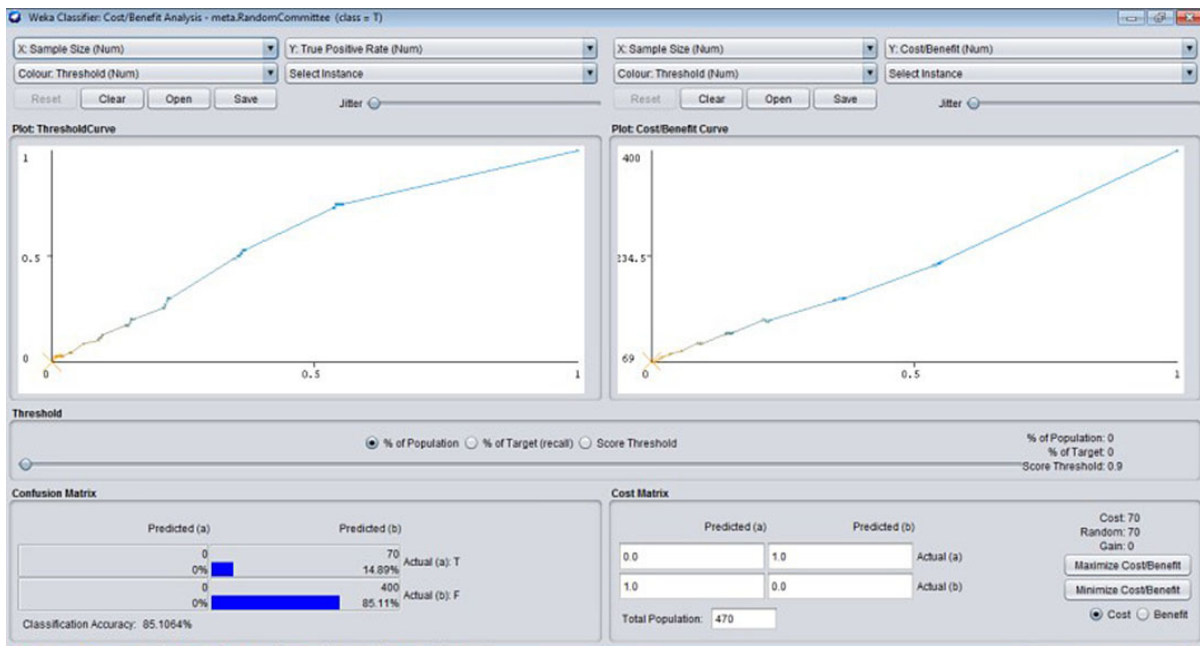

Fig. 10. Cost benefit analysis

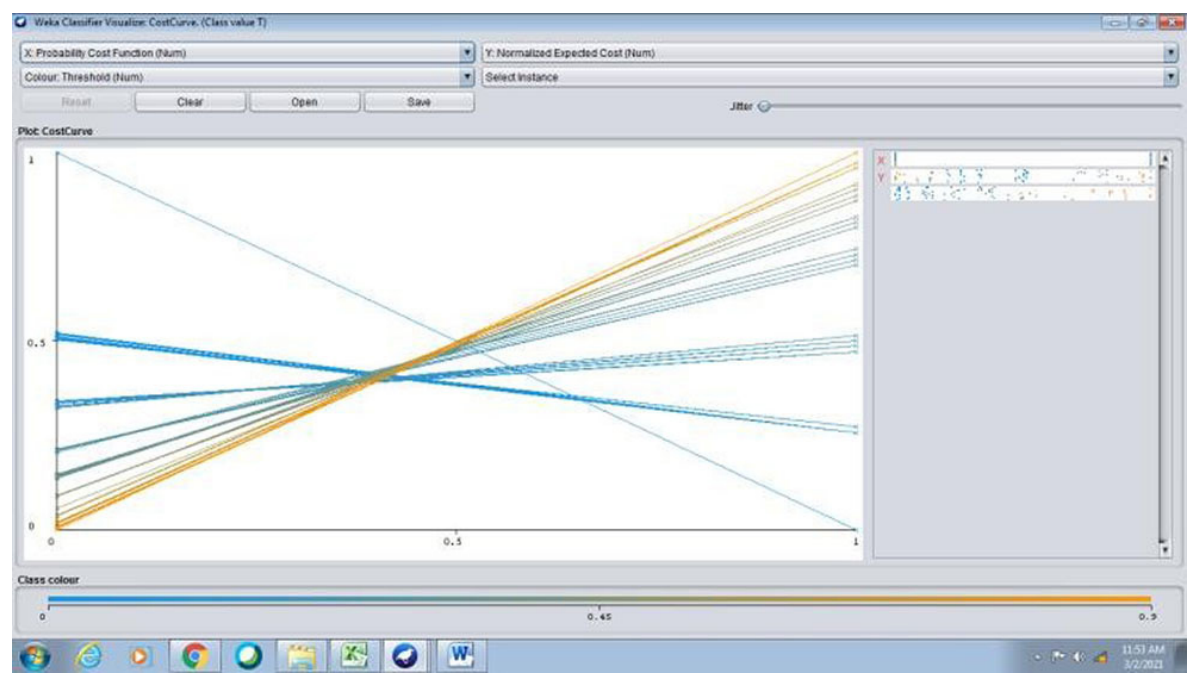

Fig. 11. Cost curve 


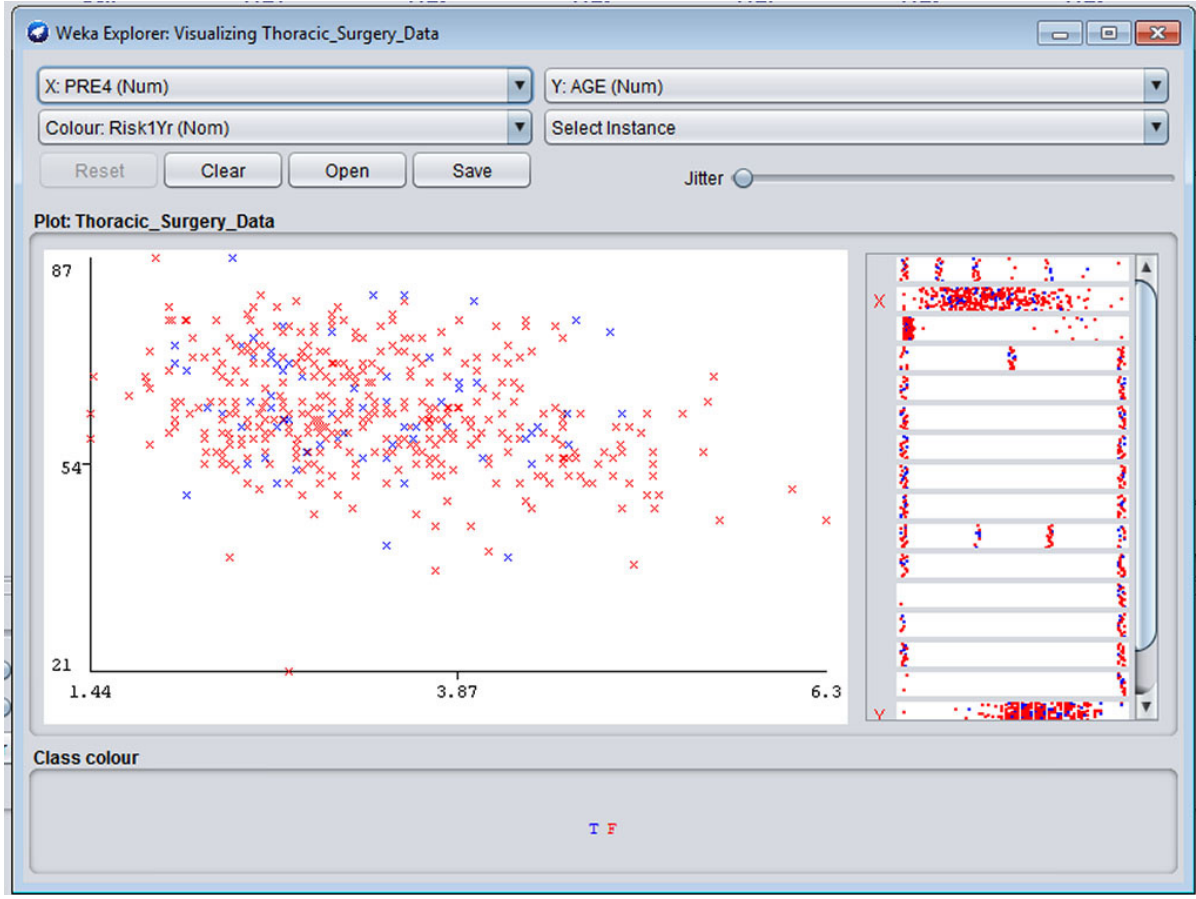

Fig. 12. Age vs. level attribute distribution graph

\section{Conclusion}

Random Committee make a bunch of specialists with changing boundaries; every now and again, these are the underlying synaptic loads, albeit different variables, (for example, the learning rate, force and so forth) might be shifted also which was stated as hypothesis statement was achieved. Random committee just like its call suggests, incorporates of infinite character choice timber that paintings as a troupe. Each person tree within the abnormal woods lets out a class forecast and the magnificence with the maximum vote's turns into our version's expectation. A greater unpredictable rendition of gathering ordinary views the give up - product no longer as a simple ordinary of the relative multitude of specialists, but as an alternative as a weighted overall.

\section{References}

[1] Gao, Y., Zhou, R., \& Lyu, Q. (2020). Multiomics and machine learning in lung cancer prognosis. Journal of Thoracic Disease, 12: 45-31. https://doi.org/10.21037/jtd-2019-itm-013

[2] Dhillon, A., Kuar, A., \& Singh, A. (2020). Application of machine learning for prediction of lung cancer using omics data. International Journal of Innovative Technology and Exploring Engineering (IJITEE), 9(6): 230-236. 
[3] Huang, H., Ma, H., \& Chen, S. (2018). Enhanced recovery after surgery using uniportal video-assisted thoracic surgery for lung cancer: A preliminary study. Thoracic Cancer, 9: 83-87. https://doi.org/10.1111/1759-7714.12541

[4] Ma, L., \& Xiang, J. (2016). Clinical outcomes of video-assisted thoracic surgery and stereotactic body radiation therapy for early-stage non-small cell lung cancer: A meta-analysis. Thoracic Cancer, 7: 442-451. https://doi.org/10.1111/1759-7714.12352

[5] Cruz, J. A., \& Wishart, D. S. (2006). Applications of machine learning in cancer prediction and prognosis. Cancer Informatics, 2: 117693510600200030. https://doi. org $/ 10.1177 / 117693510600200030$

[6] Pretorius, A., Bierman, S., \& Steel, S. J. (November 2016). A meta-analysis of research in random forests for classification. In 2016 Pattern Recognition Association of South Africa and Robotics and Mechatronics International Conference (PRASA-RobMech), November 2016, IEEE, pp. 1-6. https://doi.org/10.1109/RoboMech.2016.7813171

[7] Amer, H. M., Abou-Chadi, F. E., Kishk, S. S., \& Obayya, M. I. (2019). A CAD system for the early detection of lung nodules using computed tomography scan images. International Journal of Online \& Biomedical Engineering, 15(4). https://doi.org/10.3991/ijoe. v15i04.9837

[8] Saeed, S., Shaikh, A., Memon, M. A., Saleem, M. Q., \& Naqvi, S. M. R. (2017). Assessment of brain tumor due to the usage of MATLAB performance. Journal of Medical Imaging and Health Informatics, 7(6): 1454-1460. https://doi.org/10.1166/jmihi.2017.2187

[9] Sarkar, P., \& Chandra, V. (2020). A novel approach for detecting abnormality in ejection fraction using transthoracic echocardiography with deep learning. International Journal of Online \& Biomedical Engineering, 16(13). https://doi.org/10.3991/ijoe.v16i13.18483

[10] Saeed, S., Shaikh, A., Memon, M. A., \& Naqvi, S. M. (2018). Technique for tumor detection upon brain MRI image by utilizing support vector machine. Quaid-E-Awam University Research Journal of Engineering, Science \& Technology, Nawabshah, 16(1): 36-40.

[11] Kate, V., \& Shukla, P. (2021). Breast cancer image multi-classification using random patch aggregation and depth-wise convolution based deep-net model. International Journal of Online \& Biomedical Engineering, 17(1). https://doi.org/10.3991/ijoe.v17i01.18513

[12] Saeed, S., Shaikh, A., \& Noor, S. A. (2017). Analysis of brain tumors due to the usage of mobile phones. Mehran University Research Journal of Engineering and Technology, 36(3): 609-620. https://doi.org/10.22581/muet1982.1703.17

[13] Ahmed, S. T., \& Kadhem, S. M. (2021). Using machine learning via deep learning algorithms to diagnose the lung disease based on chest imaging: a survey. International Journal of Interactive Mobile Technologies, 15(16). https://doi.org/10.3991/ijim.v15i16.24191

[14] Shaikh, A. (2015). The impact of SOA on a system design for a telemedicine healthcare system. Network Modeling Analysis in Health Informatics and Bioinformatics, 4(1): 1-16. https://doi.org/10.1007/s13721-015-0087-0

\section{Author}

Ezzat A. Mansour, Information Science Department, Faculty of Arts and Humanities, King Abdulaziz University, Jeddah, Saudi Arabia. E-mail: ezzat556655@hotmail. com

Article submitted 2021-09-15. Resubmitted 2021-10-18. Final acceptance 2021-10-19. Final version published as submitted by the authors. 